\title{
Caracterização da movimentação de bovinos com o uso de um modelo tipo centro-periferia
}

\author{
[Characterization of the bovine movement using a core-periphery model] \\ H.O.D. Gutierrez ${ }^{1}$, E.C. Moreira ${ }^{2}$, J.A. Silva ${ }^{2}$, R.O. Rodrigues ${ }^{2}$, D.G. Vaca $^{3}$ \\ ${ }^{1}$ Servicio Nacional de Sanidad Animal e Inocuidad Alimentaria, Bolívia \\ ${ }^{2}$ Escola de Veterinária - UFMG - Belo Horizonte, MG \\ ${ }^{3}$ Dirección de Sanidad Agroalimentaria, Prefectura Departamental de Santa Cruz, Bolivia
}

\section{RESUMO}

Verificou-se a influência da movimentação de bovinos na configuração de uma estrutura do tipo centroperiferia. Foram aplicadas técnicas das redes sociais - modelo centro/periferia (C/P) contínuo - para inferir sobre a semelhança entre os padrões de movimentação registrados no estudo com uma estrutura $\mathrm{C} / \mathrm{P}$ perfeita. Os resultados mostram alta correlação, e o poder discriminatório do algoritmo permite identificar a existência de uma estrutura tipo C/P. Este resultado perfila a metodologia proposta como uma ferramenta analítica nos processos de caracterização produtiva e a regionalização de medidas de intervenção sanitária. O trabalho considera a aplicação de conceitos intuitivos sobre a definição de estruturas C/P condizentes com os determinantes de circuitos pecuários.

Palavras-chave: rede social, movimentação de bovino

\begin{abstract}
A degree in which bovine movement patterns configures a core-periphery structure was studied. Social networks analysis was used - a core-periphery (C/P) continuous model - estimating high correlation and an identification of a core with a discrimination power that outline this model as a tool for the characterization of livestock forms of production and regionalization of measures of sanitary intervention in animal health. This work considers an application of intuitive concepts about the definition of C/P structures that are according to the determinants of the livestock market circuits.
\end{abstract}

Keywords: social networks analysis, bovine movement

\section{INTRODUÇÃO}

A dimensão espacial dos processos econômicos não é circunstancial, nem cabe concebê-la como simples resultado da informação utilizada. A dimensão territorial neste aspecto é de suma importância, uma vez que as estruturas relacionais que inserem os sistemas socioeconômicos se localizam em espaços geográficos concretos.

As economias de aglomeração representam um mecanismo por meio do qual a dimensão territorial é crucial. Neste aspecto, fatores como a proximidade a recursos, mercados, concentração de fluxos (informação, produtos) representam vantagens competitivas para empresas localizadas em determinadas áreas ou cidades.

A perspectiva de redes sociais implica a aceitação de um enfoque metodológico baseado no estudo de sistemas socioeconômicos formados pelos relacionamentos entre os atores que os compõem (Semitiel e Noguera, 2004). Nestes sistemas, as relações entre os agentes constituem a base de intercâmbios, mercantis ou de outro tipo. Neste contexto, o território pode

Recebido em 19 de dezembro de 2009

Aceito em 29 de outubro de 2010

E-mail: hdaza@senasag.gov.bo 
ser entendido como um espaço que se define e se reproduz como uma rede, ou um conjunto delas, constituída por nós (ex.: instituições, empresas, organizações) entre os quais se registram fluxos que definem determinadas estruturas em evolução. Nessas estruturas, podem se achar várias posições - de poder, de dependência, de estratégia - ocupadas por instituições empresariais ou não empresariais ou, visto de outra perspectiva, pelos diversos espaços contidos na totalidade do território, por exemplo: centro e periferia.

Na década dos anos 70, estudos sobre a associação das formas de produção pecuária com doenças agudas nos animais permitiram dar razão sobre a importância de aspectos sociais e econômicos, dos seus atores envolvidos na atividade produtiva, sobre a epidemiologia das doenças: como a febre aftosa por exemplo. Em consequência disso, avanços no desenvolvimento de uma metodologia de caracterização das formas de produção pecuária, por meio do uso de indicadores epidemiológicos, seriam determinante nos processos de regionalização das medidas de intervenção sanitária (Obiaga et al., 1979).

Nesses processos de caracterização das formas de produção, uma variável associada diretamente ao risco e à disseminação de doenças no gado é a sua movimentação (Astudillo et al., 1986). As características do fluxo do trânsito de animais podem resultar da dinâmica comercial coincidentes com as formas de produção das regiões nas quais se originam. Em outras palavras, a atividade pecuária está associada a relações de abastecimento e comercialização de bens de consumo de origem animal a grandes cidades com fluxos de comercialização que influenciam na especialização regional da produção pecuária formando "circuitos" comerciais. Estes últimos surgem na base das relações de intercâmbio que se estabeleceu entre os distintos agentes que o integram no processo de produção bovina e que se relacionam entre si de forma dinâmica, influenciados por fatores socioeconômicos, proximidade geográfica, presença de mercados consumidores e valor comercial dos produtos em diferentes regiões e épocas.

As características de dependência comercial entre regiões de consumo e abastecimento, somadas à importância territorial, coincidem com a conceitualização intuitiva de Laumann e Pappi (1976) sobre as estruturas do tipo centro/periférica como áreas físicas de centro e uma periferia. Assim, pontos perto do centro de um mapa são aqueles não somente próximos entre si, mas próximos de todos os nós de um sistema ou rede; por outro lado, nós perto de áreas dos arredores ficam próximos somente dos nós do centro.

A contribuição do presente trabalho centra-se no estudo da movimentação de bovinos associando conceitos e técnicas das redes sociais condizentes com a argumentação da participação de aspectos econômicos e sociais na atividade pecuária. Diante disso, este estudo visou estimar em que medida os dados de movimentação de bovinos poderia produzir uma estrutura centro/periférica considerando os precedentes socioeconômicos em que se baseiam ambos os processos.

\section{MATERIAL E MÉTODOS}

O Departamento de Santa Cruz, principal polo industrial da Bolívia, tem experimentado, nas ultimas décadas uma especialização das formas de produção pecuária em determinadas regiões. A esperada influência dessas transformações na cadeia produtiva pecuária e, consequentemente, nos padrões de movimentação de bovinos determinou a eleição de Santa Cruz como área de estudo (Fig. 1).

Foi estimado o grau de centralidade (Wasserman e Faust, 1994) a partir do modelo proposto por Freeman (1979). O índice de centralidade de Freeman (Freeman, 1979; Hanneman e Riddle, 1995) foi calculado tanto para a rede direcionada quanto para a não direcionada. $\mathrm{O}$ modelo centro/periferia contínuo (C/P) (Borgatti e Everett, 1999) foi utilizado para inferir em que medida os padrões de movimentação de bovinos se assemelham com uma estrutura perfeita C/P.

A partir de relações entre os municípios de origem - linhas - e os de destino - colunas -, foi construída uma rede unimodal, ou seja, com um só tipo de atores e não direcionada (dados assimétricos) (Wasserman e Faust, 1994) com os dados do fluxo de bovinos. 


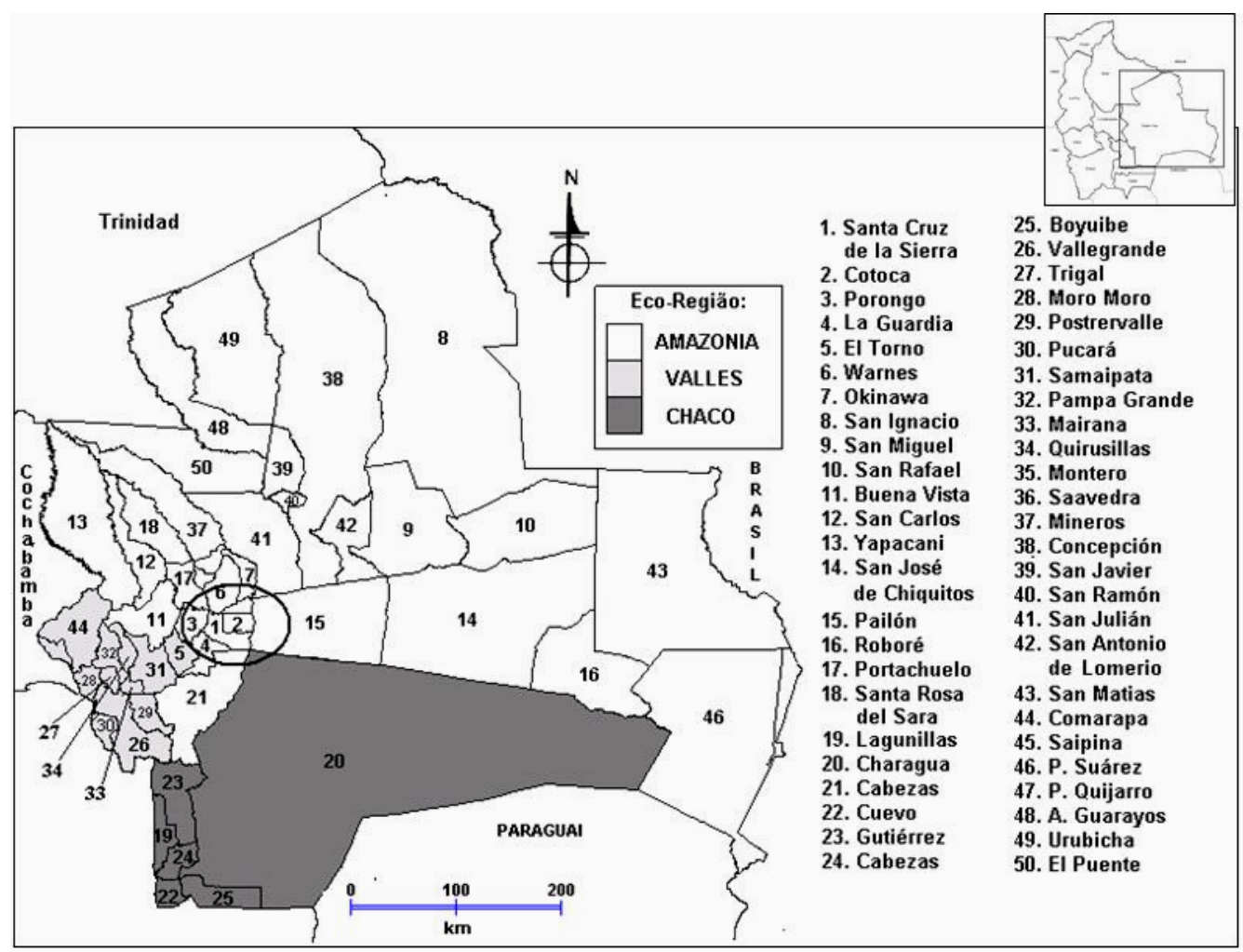

Figura 1. Ecorregiões e municípios do Departamento de Santa Cruz, Bolívia, 2008 (em círculo, polo industrial do Departamento de Santa Cruz).

No modelo contínuo para identificação de estruturas centro/periféricas, assume-se que os dados de rede consistem em dados contínuos representando poder ou capacidade relacional. Na matriz padrão, consequentemente, tem-se: a) valores elevados para pares de nós que são ambos elevados em coreness; b) valores médios para pares de nós nos quais um é elevado em coreness e o outro não; c) valores baixos para pares de nós em que ambos são periféricos. $\mathrm{O}$ modelo é consistente com a interpretação de que a largura da ligação entre dois atores é uma função de proximidade de ambos ao centro ou talvez do poder gregário de cada ator. O objetivo do algoritmo é obter valores de proximidade ao centro para calcular a correlação entre uma matriz sob estudo e uma matriz modelo para C/P. Uma característica para a definição da estrutura C/P é aquela figura com círculos concêntricos de amplitude sucessiva, centrados no centroide, onde a distância média entre pontos dentro dos círculos aumenta monotonicamente com a distância do centro. Isto significa que a intensidade da relação entre dois atores é inteiramente uma função da extensão a qual cada um é associado com o centro.

Foram estabelecidos, como atores sociais, os aglomerados de produtores pecuários existentes em cada município do Departamento de Santa Cruz. Esta unidade geopolítica coincide, na maior parte dos casos, com a área de abrangência das associações de produtores pecuaristas. Para os fins do estudo, os atores levaram o nome próprio da secção municipal ou município.

Um vínculo foi definido como a existência de movimento de bovinos entre dois municípios, relativo à movimentação de bovinos entre municípios no ano 2006. Informações sobre o trânsito de bovinos foi extraídas das guias de movimento de gado (GMG), documento oficial usado para transporte de animais dentro da República da Bolívia (Resolución..., 2001). Foi construída uma base de dados a partir das GMG emitidas no ano de 2006. O ingresso, o armazenamento, a tabulação parcial e a consulta dos dados foram realizados no programa Epi Info $^{\mathrm{TM}}$ versão 3.4.2 (Dean et al., 2007). 
Devido à importância do fluxo de bovinos procedentes de outros departamentos da Bolívia, especificamente do Departamento de Beni, foram consultados dados de posto de controle registrados no ano 2006.

A gestão de dados matriciais bem como a aplicação do modelo $\mathrm{C} / \mathrm{P}$ continuo foram executados em programa UCINET versão 6.166 (Borgatti et al., 2002). Gráficos da rede de relações de movimento de bovinos em sobreposição ao mapa da área de estudo foram realizados em programa Terraview versão 3.3.1.

\section{RESULTADOS E DISCUSSÃO}

A rede de fluxo de bovinos nos municípios do Departamento de Santa Cruz (Fig. 2) possui 387 ligações e índices Freeman de centralidade de $57,7 \%$ no global, $36,9 \%$ para graus de egresso e $61,9 \%$ para graus de ingresso. Pode-se inferir que, na rede, existe concentração de relacionamentos fazendo com que o poder dos municípios varie substancialmente, ou seja, posições de concentração de vínculos acham-se distribuídas de forma irregular na rede. Esta inferência pode ser graficamente observada na Fig. 2, onde os índices de centralidade estimam, de forma global, uma tendência mostrada na Fig. 1 para o polo industrial do Departamento de Santa Cruz, onde se acham áreas de engorda e um potencial industrial caracterizado pela existência de centros frigoríficos e abate, redes viárias estratégicas e mercados/centros de concentração de bovinos (por exemplo: centros de exposição, leilões e feiras agropecuárias).

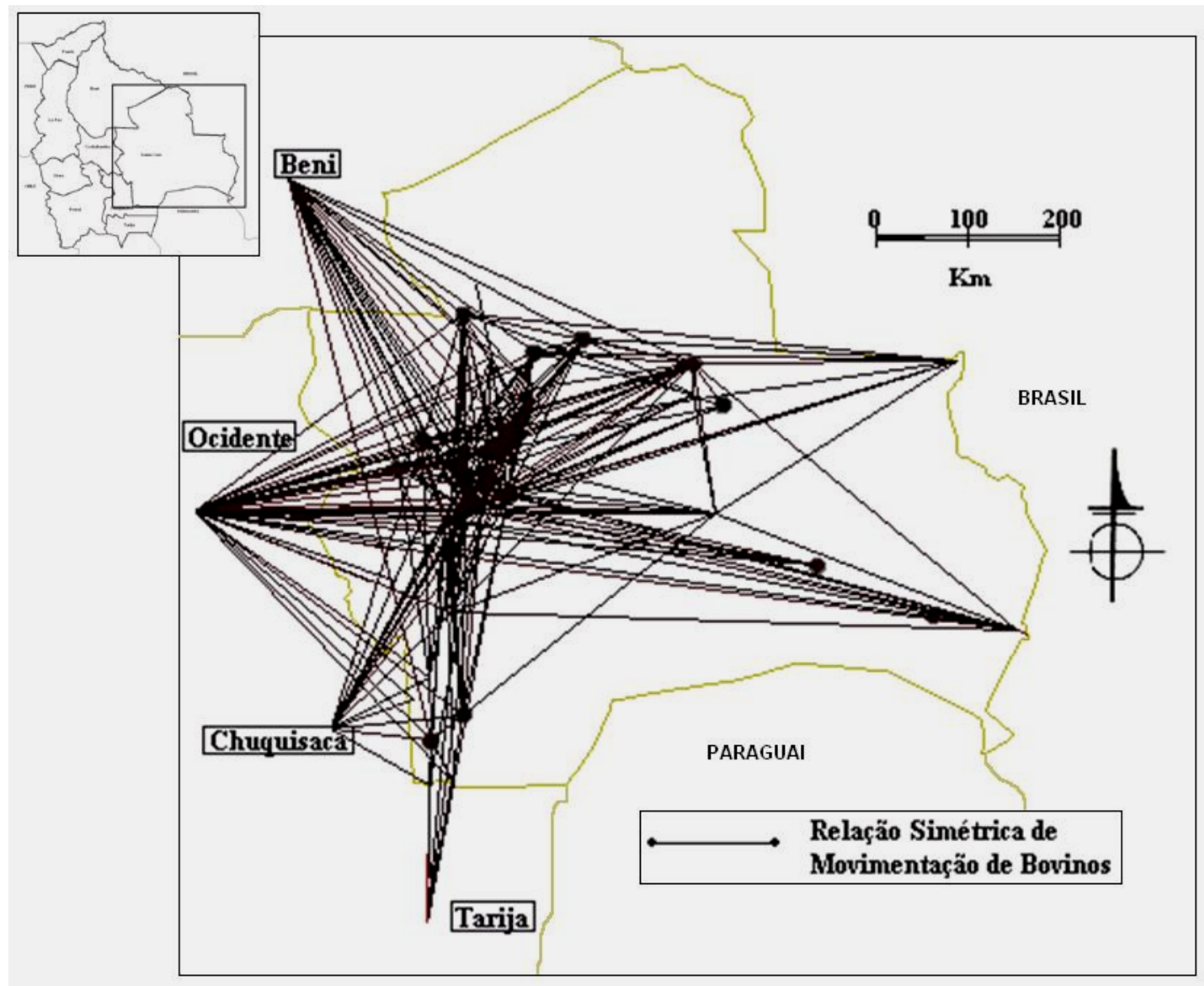

Figura 2. Rede de trânsito de bovinos intra e interdepartamental, que mostra determinadas regiões com concentração de ligações resultantes da movimentação na área central do Departamento de Santa Cruz, Bolívia, 2006. 
Este pressuposto é pormenorizado na Tab. 1, em que municípios com certo grau de centralidade "usufruem” de uma posição favorável dada pelas oportunidades de relacionamento com outros centros produtivos, permitindo-lhes certo grau de independência de outros (Hanneman e Riddle, 1995). A interpretação da centralidade, nesse sentido, proporciona conceitos homólogos aos da economia de aglomeração, na qual a dimensão territorial é crucial; fatores como proximidade a recursos, a mercados e concentração de fluxos de informação representam vantagens competitivas para as empresas localizadas em determinadas áreas ou cidades (Semitiel e Noguera, 2000).
O modelo para detecção de estruturas C/P contínuo apresenta correlação de 0,728 para os dados da rede em estudo em relação a uma estrutura C/P perfeita. Nas Fig. 2 e 3, mostram-se Santa Cruz de la Sierra, Cotoca e Pailón como os municípios recomendados pelo modelo para a composição do centro de estrutura. O indicador Coreness - grau de proximidade ao “centro” - é apresentado para cada município, junto com valores absolutos e normalizados do grau de centralidade (Tab. 1).

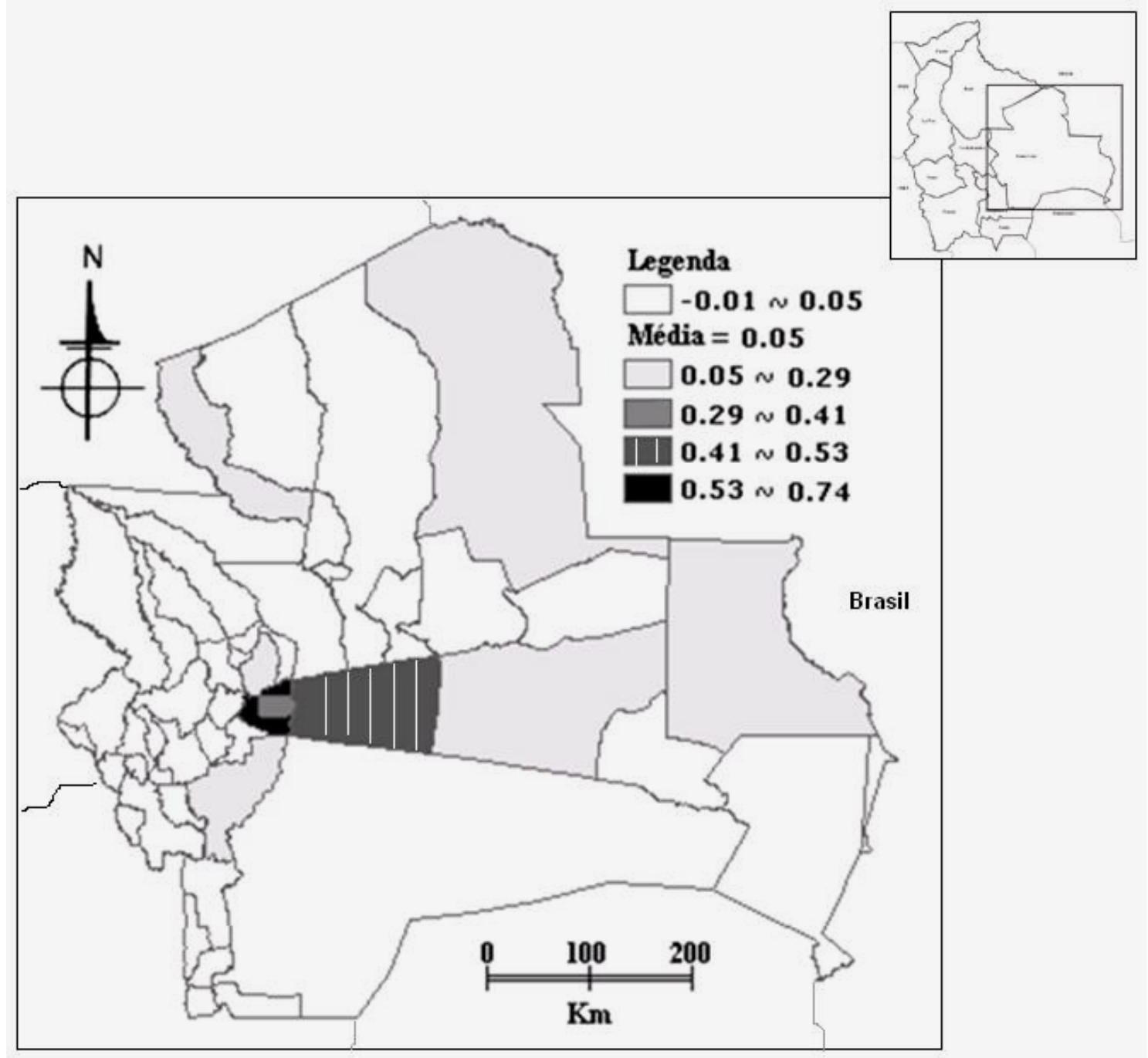

Figura 3. Grau de proximidade ao Centro ou Coreness para municípios do Departamento de Santa Cruz, resultado da analise de fluxo de movimentação de bovinos, Bolívia, 2006. 
Gutierrez et al.

Tabela 1. Grau de centralidade e Coreness para municípios do Departamento de Santa Cruz, resultado da análise de fluxo de movimentação de bovinos, Bolívia, 2006*

\begin{tabular}{|c|c|c|c|c|c|c|c|}
\hline Município & GCgl & GCglNorm & GCeg & GCegNorm & GCin & GCunNorm & Coreness \\
\hline SCZ DLS & 54 & 71,05 & 35 & 46,05 & 45 & 59,21 & 0,735 \\
\hline Pailón & 46 & 60,53 & 29 & 38,16 & 35 & 46,05 & 0,421 \\
\hline Cotoca & 46. & 60,53 & 25 & 32,9 & 38 & 50 & 0,293 \\
\hline San Ignacio & 23 & 30,26 & 22 & 28,95 & 7 & 9,21 & 0,114 \\
\hline Cabezas & 27 & 35,53 & 19 & 25 & 14 & 18,42 & 0,092 \\
\hline C.Cañadas & 25 & 32,9 & 19 & 25 & 14 & 18,42 & 0,073 \\
\hline A.Guarayos & 28 & 36,84 & 17 & 22,37 & 21 & 27,63 & 0,062 \\
\hline Warnes & 37 & 48,68 & 22 & 28,95 & 25 & 32,9 & 0,059 \\
\hline San José & 11 & 14,47 & 2 & 2,63 & 10 & 13,16 & 0,058 \\
\hline San Matías & 10 & 13,16 & 9 & 11,84 & 2 & 2,63 & 0,053 \\
\hline San Javier & 21 & 27,63 & 14 & 18,42 & 14 & 18,42 & 0,044 \\
\hline Montero & 27 & 35,53 & 14 & 18,42 & 21 & 27,63 & 0,042 \\
\hline Portachuelo & 21 & 27,63 & 13 & 17,11 & 15 & 19,74 & 0,042 \\
\hline Charágua & 14 & 18,42 & 14 & 18,42 & 3 & 3,95 & 0,040 \\
\hline San Ramón & 21 & 27,63 & 11 & 14,47 & 14 & 18,42 & 0,035 \\
\hline San Julián & 25 & 32,9 & 20 & 26,32 & 13 & 17,11 & 0,034 \\
\hline Okinawa & 21 & 27,63 & 7 & 9,21 & 17 & 22,37 & 0,029 \\
\hline Cuevo & 4 & 5,26 & 4 & 5,26 & 0 & 0 & 0,028 \\
\hline P. Suárez & 11 & 14,47 & 9 & 11,84 & 4 & 5,26 & 0,024 \\
\hline B, Vista & 7 & 9,21 & 6 & 7,9 & 3 & 3,95 & 0,022 \\
\hline San Miguel & 7 & 9,21 & 7 & 9,21 & 1 & 1,32 & 0,021 \\
\hline Mairana & 1 & 1,32 & 1 & 1,32 & 0 & 0 & 0,021 \\
\hline Camiri & 13 & 17,11 & 7 & 9,21 & 7 & 9,21 & 0,018 \\
\hline Boyuibe & 3 & 3,95 & 3 & 3,95 & 0 & 0 & 0,016 \\
\hline La Guardia & 20 & 26,32 & 3 & 3,95 & 18 & 23,68 & 0,015 \\
\hline A. Lomerio & 0 & 0 & 0 & 0 & 0 & 0 & 0,015 \\
\hline Moro Moro & 0 & 0 & 0 & 0 & 0 & 0 & 0,015 \\
\hline Gutiérrez & 3 & 3,95 & 3 & 3,95 & 0 & 0 & 0,012 \\
\hline Yapacaní & 10 & 13,16 & 3 & 3,95 & 9 & 11,84 & 0,011 \\
\hline San Carlos & 8 & 10,53 & 5 & 6,58 & 4 & 5,26 & 0,011 \\
\hline Porongo & 3 & 3,95 & 2 & 2,63 & 2 & 2,63 & 0,011 \\
\hline P. Quijarro & 1 & 1,32 & 1 & 1,32 & 0 & $\overrightarrow{0}$ & 0,010 \\
\hline Concepción & 15 & 19,74 & 13 & 17,11 & 9 & 11,84 & 0,009 \\
\hline Samaipata & 1 & 1,32 & 1 & 1,32 & 0 & 0 & 0,009 \\
\hline Postrervalle & 0 & 0 & 0 & 0 & 0 & 0 & 0,009 \\
\hline G.Saavedra & 7 & 9,21 & 7 & 9,21 & 2 & 2,63 & 0,008 \\
\hline Roboré & 6 & 7,9 & 6 & 7,9 & 1 & 1,32 & 0,008 \\
\hline Mineros & 2 & 2,63 & 2 & 2,63 & 1 & 1,32 & 0,008 \\
\hline Vallegrande & 1 & 1,32 & 1 & 1,32 & 0 & 0 & 0,008 \\
\hline Comarapa & 1 & 1,32 & 1 & 1,32 & 0 & 0 & 0,007 \\
\hline El Torno & 17 & 22,37 & 5 & 6,58 & 12 & 15,79 & 0,005 \\
\hline C. Bélgica & 3 & 3,95 & 2 & 2,63 & 1 & 1,32 & 0,005 \\
\hline Santa Rosa & 15 & 19,74 & 11 & 14,47 & 7 & 9,21 & 0,004 \\
\hline Lagunillas & 2 & 2,63 & 2 & 2,63 & 0 & 0 & 0,004 \\
\hline Urubicha & 1 & 1,32 & 1 & 1,32 & 0 & 0 & 0,002 \\
\hline Trigal & 0 & 0 & 0 & 0 & 0 & 0 & 0,002 \\
\hline El Puente & 11 & 14,47 & 8 & 10,53 & 5 & 6,58 & 0,001 \\
\hline San Rafael & 7 & 9,21 & 7 & 9,21 & 2 & 2,63 & 0,001 \\
\hline C. Rivero & 3 & 3,95 & 3 & 3,95 & 2 & 2,63 & 0,001 \\
\hline P. Grande & 1 & 1,32 & 0 & 0 & 1 & 1,32 & 0,001 \\
\hline Pucara & 0 & 0 & 0 & 0 & 0 & 0 & 0 \\
\hline Quirusillas & 0 & 0 & 0 & 0 & 0 & 0 & 0 \\
\hline Saipina & 0 & 0 & 0 & 0 & 0 & 0 & 0 \\
\hline
\end{tabular}

GC: grau de centralidade; gl: global; eg: egresso; in: ingresso; Norm: normalizado.

*Municípios ordenados segundo a participação na estrutura C/P, interpretação dada pelo indicador Coreness. — - - - Linha:Divisão dos municípios sugeridos pelo modelo como "centro" entre aqueles considerados semiperiferia e periferia. 
No contexto da América Latina, Astudillo et al. (1986) e Rosenberg (1986) explicaram que a organização espacial de cadeia produtiva de pecuária é dada em função da polarização que exerce o consumo dos centros urbanos ou os centros de saída da exportação de excedente de produção. Por esta razão, em áreas onde se desenvolvem diversas fases da produção, concentram-se núcleos de comercialização (feiras, leilões, exposições) e usinas processadoras que apresentam interações as quais pode-se interpretar como regiões favorecidas para o seu desenvolvimento empresarial.

O modelo fornece resultados que merecem consideração, por exemplo: a) evidências da existência de uma estrutura tipo C/P, uma vez que a correlação é forte $(0,72)$; b) sugestão do número de membros pertencentes ao centro; c) indicador Coreness para cada município parte da estrutura ou rede. O uso de um modelo que identifique estruturas, como a proposta por Borgatti e Everett (1999) com base em conceitos intuitivos sobre estruturas $\mathrm{C} / \mathrm{P}$ e que coincidam com os processos de formação de circuitos pecuários (Astudillo et al., 1986; Moraes, 1993), sugere sua aplicação em estudos analíticos da movimentação de bovinos como complemento aos processos de caracterização das formas de produção.

Um aspecto importante dessa análise foi considerar ligações com valores e não dicotômicas (sim/não). Segundo Borgatti e Everett (1999), a intensidade da relação entre dois atores é inteiramente uma função da extensão a qual cada ator está associado ao “centro”. Por exemplo, se considerar a mesma rede, mas com ligações dicotômicas, o poder discriminatório do modelo é influenciado, dando como resultado uma correlação de 0,517 e com 20 municípios no centro da estrutura. Dessa maneira, com o uso de dados dicotômicos, o modelo perde capacidade para discriminar municípios que, dado o conhecimento da forma de produção prevalente (por exemplo: engorda), espera-se, exerçam um papel de polo atrativo industrial, mas se se considerar vínculos com valores, o modelo define com maior precisão esse efeito desejado.

Quando se comparam o grau de centralidade e os valores coreness, Borgatti e Everett (1999) mencionaram que atores do “centro" têm necessariamente graus de centralidade elevados, enquanto o inverso não é verdade, uma vez que nem todo grupo de atores centrais fazem parte do centro. Por exemplo, é possível pegar um grupo de $n$ atores mais centrais em uma rede, de acordo com alguma medida de centralidade (ex.: grau, closeness) e, ainda, achar que o subgrafo induzido pela agrupação não contenha relação nenhuma - um centro vazio. Do ponto de vista teórico, a principal diferença entre as medidas de centralidade (como o grau de centralidade) e a medida coreness é que esta última leva consigo um modelo de padrões relacionais na rede como um todo, sendo interpretável na medida em que o modelo mantenha correlação; o grau de centralidade é interpretável independentemente da estrutura. Especial atenção merecem os municípios de San Ignácio e A. de Guarayos, em que valores elevados de coreness apontam serem possíveis subgrafos da rede inteira, havendo nesses municípios formas de produção com tendência ao ciclo completo e engorda. Assim, pode ser necessário o uso de outras técnicas das redes sociais que caracterizam sub-redes ou redes regionais/locais independentemente de uma estrutura global, por exemplo, as egorredes. Por outro lado, isto sugere que tal metodologia poderia ser usada com diferentes tipos de atores ou nós envolvidos na movimentação de bovinos (estados, municípios, escritórios de serviço veterinário, estabelecimentos pecuários). No caso de estudos no âmbito de estabelecimentos pecuários, seria determinante a disponibilidade de dados georreferenciados bem como a informação das características produtivas prevalentes no local.

\section{CONCLUSÕES}

A metodologia proposta reflete a aplicabilidade da base conceitual que o modelo $\mathrm{C} / \mathrm{P}$ contínuo pressupõe para a identificação desse tipo de estrutura em relação à exploração de dados de movimentação de bovinos, componente decisivo na caracterização das formas de produção pecuária e exercícios inerentes à regionalização de medidas em sanidade animal. Para atingir esse mesmo fim, é preciso considerar o uso de outras técnicas da esfera da análise das redes sociais dados os precedentes socioeconômicos nos quais se baseiam os processos contemplados na problemática deste trabalho: por um lado, os epidemiológicos das doenças nos animais de 
interesse econômico e, por outro, a fundamentação teórica para o uso deste tipo de algoritmo.

\section{AGRADECIMENTOS}

Ao Governo da República Federativa do Brasil, pelo apoio cedido mediante o convênio PEC-PG, e ao CNPQ (PEC-PG) - Conselho Nacional de Desenvolvimento Científico e Tecnológico - pela bolsa concedida. À Universidad Autónoma Gabriel René Moreno e à Federación de Ganaderos de Santa Cruz, (FEGASACRUZ), pela contribuição ao trabalho.

\section{REFERÊNCIAS BIBLIOGRÁFICAS}

ASTUDILLO, V.M.; DORA, J.F.P.; SILVA, A.J.M. Ecosystems and regional strategies for foot-and-mouth disease control. Application to the case of Rio Grande do Sul, Brazil. Bol. Cent. Panam. Fiebre Aftosa, n.52, p.47-77, 1986.

BORGATTI, S.P.; EVERETT, M.G. Models of core/periphery structures. Social Networks, v.21, p.375-395, 1999.

BORGATTI, S.P.; EVERETT, M.G.; FREEMAN. L.C. Ucinet 6 for Windows. Cambridge, MA: Harvard - Analytic Technologies, 2002.

DEAN, A.G.; DEAN, J.A.; BURTON, A.H. et al. EPIINFO: Database and statistics software for public health professionals. Version 3.3.2. Atlanta, 2007. Disponível em: <http://www.cdc.gov/epiinfo/>. Acessado em: 26 jan. 2008.

FREEMAN, L.C. Centrality in social networks: conceptual clarification. Social Networks, v.1, p.215-239, 1979.
HANNEMAN, R.A.; RIDDLE, M. Introduction to social network methods. Riverside. CA: University of California. Riverside, 2005. Disponível em: <http://faculty.ucr.edu/ rhanneman/>. Acessado em: 2 nov. 2007.

LAUMANN, E.O.; PAPPI, F.U. Networks of collective action: A perspective on community influence systems. New York: Academic, 1976. 329p.

OBIAGA, J.A.; ROSEMBERG, F.J.; ASTUDILLO, V.M. et al. Las caracteristicas de la produccion como determinates de los ecossistemas de fiebre aftosa. Bol. Cent. Panam. Fiebre Aftosa, n.33-34, p.33-42, 1979.

MORAES, G. The bovine commercialization circuits as elements of sanitary intervention. Bol. Cent. Panam. Fiebre Aftosa, n.59, p.29-44, 1993.

RESOLUCIÓN Administrativa del SENASAG 005/01. Art. 3. Aprueba el formato oficial del Certificado de Vacunación contra la fiebre aftosa y de la guia de movimiento de animales. Sucre, Bolívia: SENASAG, 2001.

ROSENBERG, F.J. Social structure and veterinary epidemiology in Latin America. Bol. Cent. Panam. Fiebre Aftosa, n.52, p.3-45, 1986.

SEMITIEL, G.M.; NOGUERA, M.P. Los sistemas productivos regionales desde la perspectiva del Análisis de Redes. Rev. Hisp. Anal. Redes Soc., v.6, 2004. Disponível em: $<$ http://revista-redes.rediris.es/>. Acessado em: 9 mai. 2006.

WASSERMAN, S.; FAUST, K. Social network analisys: methods and applications. Cambridge: Cambridge University, 1994. 825p. 